\title{
NON-DEGENERATE CURVES WITH MAXIMAL HARTSHORNE-RAO MODULE
}

\author{
UWE NAGEL*
}

\begin{abstract}
Extending results for space curves we establish bounds for the cohomology of a non-degenerate curve in projective $n$-space. As a consequence, for any given $n$ we determine all possible pairs $(d, g)$ where $d$ is the degree and $g$ is the (arithmetic) genus of the curve. Furthermore, we show that curves attaining our bounds always exist and describe properties of these extremal curves. In particular, we determine the HartshorneRao module, the generic initial ideal and the graded Betti numbers of an extremal curve.
\end{abstract}

\section{Contents}

\begin{tabular}{|lr}
\hline 1. & Introduction \\
\hline 2. & Bounds for the cohomology \\
\hline 3. & The possible genera of curves \\
\hline \hline.$\quad$ Construction of extremal curves & 2 \\
\hline $5 . \quad$ Properties of extremal curves & 5 \\
\hline $6 . \quad$ Betti numbers of extremal curves & 7 \\
\hline References & 9 \\
\hline
\end{tabular}

Dedicated to Silvio Greco on the occasion of his 60th birthday

\section{INTRODUCTION}

Let $\mathbb{P}^{n}$ denote the $n$-dimensional projective space over an algebraically closed field of characteristic zero. By a curve $C \subset \mathbb{P}^{n}$ we will always understand a closed subscheme which is locally Cohen-Macaulay of pure dimension 1, thus in particular without isolated or embedded points. The curve is called non-degenerate if it is not contained in a hyperplane of $\mathbb{P}^{n}$. The most important invariants of the curve $C$ are its degree and its (arithmetic) genus. However, a more precise description of $C$ requires some knowledge of its Hartshorne-Rao module. Its importance is particularly highlighted in Liaison theory (cf. [19]) and in the classification theory of curves (cf. [15]).

Every graded module of finite length is (up to degree shift) the Hartshorne-Rao module of a curve (cf. [21]). However, fixing the degree $d$ and the (arithmetic) genus $g$ puts restrictions on the possible Hartshorne-Rao modules. In this paper we mainly investigate the restrictions on the "size" of the Hartshorne-Rao module. More precisely, we consider the following basic problems.

Problems. Let $C \subset \mathbb{P}^{n}$ be a non-degenerate curve of degree $d$ and (arithmetic) genus $g$. (1) Which pairs $(d, g)$ can occur?

* Department of Mathematics, University of Kentucky, Lexington, KY 40506-0027, USA e-mail: uwenagel@ms.uky.edu. 
(2) Find (optimal) upper estimates for the dimension $h^{1}\left(\mathcal{I}_{C}(j)\right), j \in \mathbb{Z}$.

(3) Are there curves attaining the bounds in (2) for all $j \in \mathbb{Z}$ ?

The answers to these problems are completely known for space curves, i.e. when $n=3$. The goal of this paper is to answer these questions for arbitrary $n \geq 3$ and to describe properties of the extremal curves occurring in Problem (3). Hereby we are using tools from cohomology theory, homological algebra as well as the theory of Gröbner bases. On a technical level one problem is to replace codimension two oriented methods by those which work in any codimension. Some of the results in this note have been announced in 24.

The problems above, in particular Problem (1), are very classical for irreducible, reduced curves. However, we consider them for more general curves because even if one is primarily interested in integral curves one often is led to study more generally locally Cohen-Macaulay curves. This is evident, for example, in Liaison theory or in the study of families of curves. For the latter the Hilbert scheme $H_{d, q}^{n}$ of locally Cohen-Macaulay curves in $\mathbb{P}^{n}$ of degree $d$ and genus $g$ is the right framework. Of course, the first problem above just asks: When does the Hilbert scheme $H_{d, g}^{n}$ contain a non-degenerate curve?

Let us describe the organization of the paper. Bounds for the first and second cohomology of the ideal sheaf of a curve are established in Section 2. These results lead immediately to a bound on the genus depending on the degree. In Section 3 we show that this is the only restriction on the genus by constructing suitable curves. A refinement of the construction allows to construct curves attaining the bound on the Hilbert function of the Hartshorne-Rao module in every degree. This is done in Section 4 and the resulting curves are called extremal curves.

The remaining parts of this note are devoted to study these curves. It turns out that the situation for space curves is more rigid and simpler than for curves of codimension $\geq 3$. In Section 5 we describe the structure of the Hartshorne-Rao module of an extremal curve. A key is the determination of the generic initial ideal of an extremal curve. Furthermore, we show that extremal curves also have maximal second cohomology and (mostly) contain a planar subcurve of degree $d-1$. The converse to these two results ist only true if $n=3$.

In Section 6 we compute the graded Betti numbers of an extremal curve. As a consequence we get that its homogeneous ideal is componentwise linear if the degree of the curve is at least 5 .

The results in [17] strongly suggest that it is worth to investigate families of extremal curves using the results of Section 5 and 6 . We wish to pursue this in a future paper.

\section{BOUndS FOR THE COHOMOLOGY}

In this section we establish estimates for the first and second cohomology of a curve $C$. Note that in [5], Theorem 2.1 such bounds have been achieved under the additional assumption that the general hyperplane section of $C$ in non-degenerate.

If a curve of degree 2 is not arithmetically Cohen-Macaulay then it is a pair of two skew lines or a double line. Thanks to the results in [22] we have a complete description of all double lines. Thus, in this note we often will focus on curves of degree $d \geq 3$.

Proposition 2.1. Let $C \subset \mathbb{P}^{n}$ be a non-degenerate curve of degree $d \geq 3$ and (arithmetic) genus $g$. Then we have:

(a)

$$
h^{1}\left(I_{C}(j)\right) \leq \rho_{n}^{e x}(j) \quad \text { for all } j \in \mathbb{Z}
$$


where $\rho_{n}^{e x}: \mathbb{Z} \rightarrow \mathbb{Z}$ is the function defined by

$$
\rho_{n}^{e x}(j)= \begin{cases}0 & \text { if } j \leq-\left(\begin{array}{c}
d-2 \\
2
\end{array}\right)+g \\
\left(\begin{array}{c}
d-2 \\
2
\end{array}\right)-g+j & \text { if } \quad-\left(\begin{array}{c}
d-2 \\
2
\end{array}\right)+g \leq j \leq 0 \\
\left(\begin{array}{c}
d-2 \\
2
\end{array}\right)-g-(n-3) & \text { if } 1 \leq j \leq d-2 \\
\left(\begin{array}{c}
d-1 \\
2
\end{array}\right)-g-(n-3)-j & \text { if } \quad d-2 \leq j \leq\left(\begin{array}{c}
d-1 \\
2
\end{array}\right)-g-(n-3) \\
0 & \text { if } \quad\left(\begin{array}{c}
d-1 \\
2
\end{array}\right)-g-(n-3) \leq j\end{cases}
$$

(b)

$$
h^{2}\left(\mathcal{I}_{C}(j)\right) \leq \mu_{n}^{e x}(j) \quad \text { for all } j \in \mathbb{Z}
$$

where $\mu_{n}^{e x}: \mathbb{Z} \rightarrow \mathbb{Z}$ is the function defined by

$$
\mu_{n}^{e x}(j)= \begin{cases}0 & \text { if } j \geq d-3 \\
\left(\begin{array}{c}
d-2-j \\
2
\end{array}\right) & \text { if } 0 \leq j \leq d-2 \\
\left(\begin{array}{c}
d-2 \\
2
\end{array}\right)-(d-1) j-1 & \text { if } g-\left(\begin{array}{c}
d-2 \\
2
\end{array}\right) \leq j \leq-1 \\
g-1-d j & \text { if } j \leq g-\left(\begin{array}{c}
d-2 \\
2
\end{array}\right)-1 .\end{cases}
$$

The strategy of proof is similar to the one for Theorem 2.1 in [5]. Thus, we will focus on the differences and refer to [5] for more details.

Throughout the paper we will denote by $R:=K\left[x_{0}, \ldots, x_{n}\right]$ the homogeneous coordinate ring of $\mathbb{P}^{n}$. Let $\Gamma:=C \cap H$ be the general hyperplane section of $C$. While $C \subset \mathbb{P}^{n}$ is non-degenerate $\Gamma \subset H \cong \mathbb{P}^{n-1}$ might be degenerate. The first step consists in estimating the failure of non-degeneracy of $\Gamma$. It strengthens [2], Proposition 4.2.

Lemma 2.2. Let $C \subset \mathbb{P}^{n}, n \geq 3$, be a non-degenerate curve of degree $d \geq 3$. Then its general hyperplane section $\Gamma$ is not collinear.

Proof. Let $\ell \in R$ be the linear form defining the general hyperplane $H$. Put $m:=$ $h^{0}\left(\mathcal{I}_{\Gamma}(1)\right)$. If $m=0$ there is nothing to show. Assume $m \geq 1$. Let $f_{1}, \ldots, f_{m}$ be linearly independent linear forms in the homogeneous ideal $I_{\Gamma}$ of $\Gamma \subset H$. Then $T:=$ $\bar{R} /\left(\ell, f_{1}, \ldots, f_{m}\right)$ is the homogeneous coordinate ring of the linear span $L$ of $\Gamma$. Let $\mathfrak{c}:=I_{\Gamma} T$ be the ideal of $\Gamma \subset L$. Using the so-called Socle lemma of Huneke and Ulrich (cf. [14]) it is shown in Steps 1 and 3 of the proof of [5], Theorem 1.4 that the ideal $\mathfrak{c}$ has codimension $\leq n-1-m$ and contains at least $n-1-m$ linearly independent quadrics. It follows that $m \leq n-2$. We have to exclude the possibility $m=n-2$. Indeed, if $m=n-2$ then we obtain $\operatorname{deg} C=\operatorname{deg} \Gamma=\operatorname{deg} \mathfrak{c} \leq 2$. This contradiction completes the proof.

Remark 2.3. The assumption on the characteristic of the ground field is used for the application of the Socle lemma. In fact, the statement above is not true over fields of positive characteristic. There are multiple lines in $\mathbb{P}^{3}$ whose general hyperplane section is collinear (cf. [13]).

The second step in the proof of Proposition 2.1 is mainly an application of the methods developed in [3], [23] and [4]. For details we refer to the proof of [5], Theorem 2.1. 
Proof of Proposition 2.1. We proceed in several steps.

Notation. We use $h_{C}$ and $p_{C}\left(\operatorname{resp} . h_{\Gamma}\right.$ and $\left.p_{\Gamma}\right)$ to denote the Hilbert function and the Hilbert polynomial of $C$ (resp. of $\Gamma$ ).

Step 1. According to Lemma 2.2 we have $h_{\Gamma}(1) \geq 3$, thus $h^{1}\left(\mathcal{I}_{\Gamma}(1)\right) \leq d-3$. Therefore Lemma 4.3 of [23] provides for $j \geq 1$ :

$$
h^{1}\left(\mathcal{I}_{\Gamma}(j)\right) \leq \max \{0, d-2-j\}
$$

and

$$
h_{\Gamma}(j) \geq \min \{d, 2+j\}
$$

Step 2. The long exact cohomology sequence induced by the exact sequence

$$
0 \rightarrow \mathcal{I}_{C}(j-1) \rightarrow \mathcal{I}_{C}(j) \rightarrow \mathcal{I}_{\Gamma}(j) \rightarrow 0
$$

ends as follows:

$$
\cdots \rightarrow H^{1}\left(\mathcal{I}_{\Gamma}(j)\right) \rightarrow H^{2}\left(\mathcal{I}_{C}(j-1)\right) \rightarrow H^{2}\left(\mathcal{I}_{C}(j)\right) \rightarrow 0
$$

Since $H^{2}\left(I_{C}(j)\right)=0$ for $j \gg 0$, we get by Step 1 if $j \geq 0$ :

$$
\begin{aligned}
h^{2}\left(\mathcal{I}_{C}(j)\right) & \leq \sum_{t \geq j+1} h^{1}\left(\mathcal{I}_{\Gamma}(t)\right) \\
& \leq \sum_{t \geq j+1} \max \{0, d-2-t\} \\
& =\left(\begin{array}{c}
d-2-j \\
2
\end{array}\right)
\end{aligned}
$$

proving claim (b) for non-negative degrees.

Step 3. Consider now the following version of the Riemann-Roch theorem:

$$
h_{C}(j)-p_{C}(j)=-h^{1}\left(I_{C}(j)\right)+h^{2}\left(I_{C}(j)\right) \quad(j \in \mathbb{Z})
$$

where $p_{C}(j)=d j-g+1$.

Using Step 2 and $h_{C}(1)=n+1$ we obtain for $j \geq 1$ :

$$
\begin{aligned}
h^{1}\left(\mathcal{I}_{C}(j)\right)= & p_{C}(j)-h_{C}(j)+h^{2}\left(\mathcal{I}_{C}(j)\right) \\
\leq & d j-g+1-\left[n+1+\sum_{t=2}^{j} h_{\Gamma}(t)\right]+ \\
& +\sum_{t \geq j+1} \max \{0, d-2-t\} \quad(\text { by Step 2) } \\
\leq & \left.d-g-n+\sum_{t \geq 2} \max \{0, d-2-t\} \quad \text { (by Step } 1\right) \\
= & \left(\begin{array}{c}
d-2 \\
2
\end{array}\right)-g+3-n .
\end{aligned}
$$

For $j=0$ we get

$$
h^{1}\left(\mathcal{I}_{C}\right) \leq-g+\left(\begin{array}{c}
d-2 \\
2
\end{array}\right)
$$


This proves claim (a) if $0 \leq j \leq d-2$. For other values of $j$ the result follows now by the general methods developed in [4] and [23] (cf. Steps 4 and 5 in the proof of [5], Theorem 2.1).

Step 4. For $j<0$ the Riemann-Roch theorem implies

$$
h^{2}\left(\mathcal{I}_{C}(j)\right)=h^{1}\left(\mathcal{I}_{C}(j)\right)-[d j-g+1] .
$$

Thus, in this case claim (b) follows by (a). Together with Step 2 this completes the proof.

Observe that part (a) of Proposition 2.1 generalizes the estimates of Martin-Deschamps and Perrin in [16] from $n=3$ to $n \geq 3$ while part (b) extends [7], Lemma 4.8.

In Proposition 2.1 we have defined the function $\rho_{n}^{e x}$ for curves of degree $d \geq 3$. We want to extend it to curves of degree 2 .

Definition 2.4. If $d=2$ we set

$$
\rho_{n}^{e x}(j):= \begin{cases}0 & \text { if } j \leq g \\ -g+j & \text { if } g \leq j \leq 0 \\ -g-(n-3)-j & \text { if } 1 \leq j \leq-g-(n-3) \\ 0 & \text { if }-g-(n-3) \leq j\end{cases}
$$

Using this notation we can generalize Proposition 2.1

Theorem 2.5. Let $C \subset \mathbb{P}^{n}, n \geq 3$, be a non-degenerate curve of degree $d$ and (arithmetic) genus $g$. Then we have:

$$
h^{1}\left(I_{C}(j)\right) \leq \rho_{n}^{e x}(j) \quad \text { for all } j \in \mathbb{Z} .
$$

Proof. Since the curve $C$ is non-degenerate its degree must be at least two. Thus, by virtue of Proposition 2.1 it remains to consider the case $d=2$. If $C$ is arithmetically Cohen-Macaulay then it is a planar conic contradicting our assumption. Hence, $C$ is not arithmetically Cohen-Macaulay, thus it must be a pair of two skew lines or a double line. Therefore, the claim follows by [22], Corollary 3.2.

The objective of the next two sections is to show that the bounds in the theorem above cannot be improved.

\section{The Possible GenerA of CURVES}

Theorem 2.5 immediately implies.

Corollary 3.1. Let $C \subset \mathbb{P}^{n}$ be a non-degenerate curve of degree $d \geq 3$. Then the arithmetic genus $g$ of $C$ satisfies

$$
g \leq\left(\begin{array}{c}
d-2 \\
2
\end{array}\right)-(n-3)
$$

Here we want to show that this is the only restriction. We also include curves of degree 2. Notice, that in this case [22], Corollary 2.10 provides $g \leq 2-n$.

Theorem 3.2. A non-degenerate curve $C \subset \mathbb{P}^{n}, n \geq 3$, of degree $d \geq 2$ can have every genus $g \leq g_{\max }:=\left\{\begin{array}{cl}\left(\begin{array}{c}d-2 \\ 2\end{array}\right)-(n-3) & \text { if } d \geq 3 \\ 2-n & \text { if } d=2 .\end{array}\right.$ 
We will show this result by constructing suitable curves. We use ideas of the proof of Proposition 1.11 in [5] where the statement has been shown in case $n=4$. For $n=3$ the result has been proved independently in [29], [27] and [13].

Construction 3.3. Let $D \subset \mathbb{P}^{n}, n \geq 3$, be a planar curve of degree $d-1$ which is supported on the line $L$. Let $a \geq 0$ be an integer.

We may assume that the homogeneous ideals of $L$ and $D$ in $R=K\left[x_{0}, \ldots, x_{n}\right]$ are $I_{L}=\left(x_{2}, \ldots, x_{n}\right)$ and $I_{D}=\left(x_{2}^{d-1}, x_{3}, \ldots, x_{n}\right)$, respectively. Let $S=K\left[x_{0}, x_{1}\right] \cong R / I_{L}$ be the homogeneous coordinate ring of $L$. Observe that there is an isomorphism of graded $S$-modules

$$
I_{D} / I_{L} I_{D} \cong S^{n-2}(-1) \oplus S(-d+1) .
$$

Now we choose homogeneous elements $f_{1}, \ldots, f_{n-2}, f \in S$ such that $f_{1}, \ldots, f_{n-2}$ have degree $a+n-3$ and $f$ has degree $d+a+n-5$. Notice that our assumptions imply $a+n-3 \geq 0$ and $d+a+n-5 \geq 0$. Using these forms we define the following homomorphism

$$
\psi: S^{n-2}(-1) \oplus S(-d+1) \stackrel{\left(f_{1}, \ldots, f_{n-2}, f\right)}{\longrightarrow} S(a+n-4) .
$$

Let $\alpha: I_{D} \rightarrow S(a+n-4)$ be the composition

$$
I_{D} \rightarrow I_{D} / I_{L} I_{D} \stackrel{\cong}{\longrightarrow} S^{n-2}(-1) \oplus S(-d+1) \stackrel{\psi}{\longrightarrow} S(a+n-4)
$$

where the first map is the canonical epimorphism. Since $D$ and $L$ are arithmetically Cohen-Macaulay ker $\alpha \subset I_{D}$ is a homogeneous saturated ideal defining a 1-dimensional subscheme $C \subset \mathbb{P}^{n}$. Moreover, we have an exact sequence of graded $R$-modules

$$
0 \rightarrow I_{C} \rightarrow I_{D} \stackrel{\alpha}{\longrightarrow} R / I_{L}(a+n-4) \rightarrow \operatorname{coker} \alpha \rightarrow 0 \text {. }
$$

Keeping the notation and assumptions of the construction we get.

Lemma 3.4. Assume that coker $\alpha$ has finite length, $d \geq 3$ and that $f_{1}, \ldots, f_{n-2}$ are linearly independent. Then there is a non-degenerate curve $C \subset \mathbb{P}^{n}$ fitting into the exact sequence

$$
0 \rightarrow \mathcal{O}_{L}(a+n-4) \rightarrow \mathcal{O}_{C} \rightarrow \mathcal{O}_{D} \rightarrow 0
$$

Proof. Since coker $\alpha$ has finite length, sheafification of the exact sequence $(*)$ above provides the exact sequences

$$
0 \rightarrow \mathcal{I}_{C} \rightarrow \mathcal{I}_{D} \rightarrow \mathcal{O}_{L}(a+n-4) \rightarrow 0
$$

and

$$
0 \rightarrow \mathcal{O}_{L}(a+n-4) \rightarrow \mathcal{O}_{C} \rightarrow \mathcal{O}_{D} \rightarrow 0 \text {. }
$$

Clearly, the support of $C$ is $L$. Furthermore, the second sequence shows for all closed points $P \in L$ that $\mathcal{O}_{C, P}$ has depth 1 . Hence is $C$ is a locally Cohen-Macaulay subscheme of pure dimension 1, i.e. a curve.

Since $\operatorname{ker} \alpha$ is the homogeneous ideal of $C$, the curve $C$ is non-degenerate if and only if $[\operatorname{ker} \psi]_{1}=0$. But this just means that $f_{1}, \ldots, f_{n-2}$ are linearly independent because $[S(-d+1)]_{1}=0$.

Now we can show Theorem 3.2 easily.

Proof of Theorem 3.9. If $d=2$ the claim follows by [22], Theorem 2.6 and Proposition 2.9 .

Assume $d \geq 3$. Of course, we want to apply the lemma above. We have to check that we can satisfy its assumptions. But $[S]_{a+n-3}$ is a $K$-vector space of dimension $a+n-2 \geq n-2$. Thus we can find linearly independent elements $f_{1}, \ldots, f_{n-2}$ in $[S]_{a+n-3}$. Moreover, we 
can certainly arrange that coker $\psi$ and thus coker $\alpha$ has finite length. For example, choose $f_{1}=x_{0}^{a+n-3}$ and $f=x_{1}^{d+a+n-5}$.

Now using the sequence in Lemma 3.4 an easy computation of Hilbert polynomial shows that $C$ is a curve of degree $d$ and arithmetic genus $g_{\max }-a$. Since $a$ is any non-negative integer we are done.

Remark 3.5. An analysis of the construction above shows that in order to prove Theorem 3.2 we could even construct a curve $C$ having the two additional properties:

(a) $C$ has generically embedding dimension 2 (in particular it is a generically complete intersection);

(b) $C$ is irreducible, i.e. it is not the scheme-theoretic union of any two closed subschemes of $C$, different from $C$.

This follows as in the proof of [5], Proposition 1.11. The reader interested in seeing such a curve described by explicit equations is referred to Example 4.5 .

\section{Construction of extremal Curves}

Following Martin-Deschamps and Perrin we define.

Definition 4.1. A non-degenerate curve $C \subset \mathbb{P}^{n}$ is called extremal if

$$
h^{1}\left(\mathcal{I}_{C}(j)\right)=\rho_{n}^{e x}(j) \quad \text { for all } j \in \mathbb{Z} .
$$

A priori it is not clear at all that such extremal curves do exist. In case $n=3$ the existence has been established by Martin-Deschamps and Perrin ([16]). A first generalization of this result to $n \geq 4$ has been achieved in [5] where it was assumed that with $C$ also its general hyperplane section is non-degenerate. Here we want to generalize the result for $n=3$ to $n \geq 3$ without assuming additional properties. This is taken care of by refining the construction of the previous section.

Theorem 4.2. For every pair $(d, g)$ of integers such that $d \geq 2$ and $g \leq g_{\text {max }}$ there is an extremal curve $C \subset \mathbb{P}^{n}$.

Remark 4.3. Contrary to the situation for space curves, extremal curves of $\mathbb{P}^{n}$ of maximal genus $g=g_{\max }$ are not arithmetically Cohen-Macaulay if $n \geq 4$.

Much of this section is based on the following observation concerning Construction 3.3.

Lemma 4.4. With the notation and assumptions of Construction 3.3 assume additionally that coker $\alpha$ has finite length. Then the scheme $C$ constructed there is a curve with Hartshorne-Rao module

$$
H_{*}^{1}\left(\mathcal{I}_{C}\right) \cong\left(R /\left(I_{L}+\left(f_{1}, \ldots, f_{n-2}, f\right) R\right)\right)(a+n-4) .
$$

Proof. First of all note that

$$
\text { coker } \alpha=\text { coker } \psi \cong\left(R /\left(I_{L}+\left(f_{1}, \ldots, f_{n-2}, f\right) R\right)\right)(a+n-4) \text {. }
$$

The exact sequence $(*)$ in Construction 3.3 provides the exact sequences

$$
0 \rightarrow I_{C} \rightarrow I_{D} \rightarrow \operatorname{im} \alpha \rightarrow 0
$$

and

$$
0 \rightarrow \operatorname{im} \alpha \rightarrow R / I_{L}(a+n-4) \rightarrow \operatorname{coker} \alpha \rightarrow 0 .
$$

Since $R / I_{L}$ is Cohen-Macaulay the second sequence implies

$$
H_{\mathfrak{m}}^{1}(\operatorname{im} \alpha) \cong H_{\mathfrak{m}}^{0}(\operatorname{coker} \alpha) \cong \operatorname{coker} \alpha
$$


Using the first sequence and that $D$ is arithmetically Cohen-Macaulay we obtain

$$
H_{\mathfrak{m}}^{2}\left(I_{C}\right) \cong H_{\mathfrak{m}}^{1}(\operatorname{im} \alpha)
$$

Taking into account the isomorphism

$$
H_{*}^{1}\left(\mathcal{I}_{C}\right) \cong H_{\mathfrak{m}}^{2}\left(I_{C}\right)
$$

the claim follows.

This lemma reduces the proof of Theorem 4.2 to a suitable choice of the polynomials $f_{1}, \ldots, f_{n-2}, f$.

Proof of Theorem 4.9. If $d=2$ the claim follows by [22], Corollary 3.2.

Assume $d \geq 3$. Let $h \neq 0$ be a homogeneous polynomial of degree $a:=g_{\max }-g \geq 0$ in $S=k\left[x_{0}, x_{1}\right]$. Then we choose $f_{1}, \ldots, f_{n-2} \in[S]_{a+n-3}$ such that

$$
\left(f_{1}, \ldots, f_{n-2}\right)=h\left(x_{0}, x_{1}\right)^{n-3} .
$$

Finally, we choose $f \in[S]_{d+a+n-5}$ such that $\{f, h\}$ is a regular sequence unless $a=0$ and $n=3$. (If $a=0$ there is no condition of $f$.)

Then it is easy to see that we have for all integers $j \in \mathbb{Z}$

$$
\operatorname{rank}_{K}\left[R /\left(I_{L}+\left(f_{1}, \ldots, f_{n-2}, f\right)\right]_{j+a+n-4}=\rho_{n}^{e x}(j) .\right.
$$

Therefore Lemma 4.4 and Lemma 3.4 show that $C$ is an extremal curve.

For each pair $(d, g)$ allowed by Theorem 4.2 we describe one extremal curve by explicit equations.

Example 4.5. In the last proof we specialize the polynomials $f, h$ to

$$
f:=x_{1}^{d+a+n-5} \text { and } h:=x_{0}^{a}
$$

where $a:=g_{\max }-g$. Then, it is not too difficult to see that $\operatorname{ker} \alpha$ is the ideal

$$
\begin{aligned}
I=\left(x_{2}^{d-1}, x_{3}, \ldots, x_{n}\right) \cdot\left(x_{2}, \ldots, x_{n}\right)+ \\
\\
\quad\left(x_{0}^{a} x_{2}^{d-1}+x_{1}^{d+a-2} x_{3}, x_{0} x_{i}+x_{1} x_{i+1} \mid 3 \leq i \leq n-1\right) .
\end{aligned}
$$

Hence $I$ defines an extremal curve $C \subset \mathbb{P}^{n}$ of degree $d$ and genus $g=g_{\text {max }}-a$ provided $d \geq 3$. If $d=2$ and $a>0$ then the ideal $I$ defines an extremal curve as well. It has genus $3-n-a$. Notice that in all cases this extremal curve is irreducible and has generically embedding dimension 2 .

We want to point out that the situation is more rigid for $n=3$ than for $n \geq 4$. Indeed, it follows by 25 that a non-degenerate curve $C \subset \mathbb{P}^{3}$ of degree $d \geq 4$ with $h^{1}\left(\mathcal{I}_{C}(1)\right)=\rho_{3}^{e x}(1)>0$ must be an extremal curve. The example below shows that the analogous conclusion is not true if $n \geq 4$. It is even false if we assume $h^{1}\left(\mathcal{I}_{C}(j)\right)=\rho_{3}^{e x}(j)$ for all $j \leq 1$.

Example 4.6. We use again the Construction 3.3. Suppose that $n \geq 4$ and $a>0$. Define integers $k, \varepsilon$ by

$$
a=:(k-1)(n-3)+\varepsilon \quad \text { where } 0 \leq \varepsilon \leq n-4 .
$$

Put $b=:(n-2)(a+n-3)$ and define the polynomials $f_{1}, \ldots, f_{n-2}$ by

$$
\left(f_{1}, \ldots, f_{n-2}\right)=\left(x_{0}^{b}, x_{0}^{i k} x_{1}^{(n-3-i) k+\varepsilon} \mid 0 \leq i \leq n-4\right) .
$$


Setting $f:=0$ (which is allowed!) we obtain by Lemma 3.4 a non-degenerate curve $C \subset \mathbb{P}^{n}$ of degree $d$ and genus $g_{\max }-a$ whose Hartshorne-Rao module is given by Lemma 4.4. Let $d \geq 4$. Then we get

$$
h^{1}\left(\mathcal{I}_{C}(j)\right)=\rho_{3}^{e x}(j) \quad \text { for all } j \leq 1
$$

and

$$
h^{1}\left(\mathcal{I}_{C}(2)\right)<h^{1}\left(\mathcal{I}_{C}(1)\right)=a=\rho_{n}^{e x}(1)=\rho_{n}^{e x}(2) .
$$

Hence $C$ is not an extremal curve.

Remark 4.7. We have seen that the Construction 3.3 produces a non-degenerate curve of degree $d \geq 3$ and genus $g_{\max }-a$ for all choices of linearly independent forms $f_{1}, \ldots, f_{n-2} \in$ $S$ of degree $a+n-3$ and a form $f \in S$ of degree $d+a+n-5$ such that $S /\left(f, f_{1}, \ldots, f_{n-2}\right) S$ has finite length. Then Lemma 4.4 gives for the resulting curve $C$ that $H_{*}^{1}\left(\mathcal{I}_{C}\right) \cong$ $\left(S /\left(f, f_{1}, \ldots, f_{n-2}\right) S\right)(a+n-4)$. Thus, it is not too difficult to determine the possible Rao functions of the curves obtained by Construction 3.3. In particular, every such curve $C \subset \mathbb{P}^{n}$ satisfies

$$
h^{1}\left(\mathcal{I}_{C}(j)\right)=\rho_{n}^{e x}(j) \quad \text { for all } j \leq 1
$$

The next remark shows that the situation in case $d=3$ is somewhat particular.

Remark 4.8. We will see in the next section that Lemma 4.4 describes the HartshorneRao module of every extremal curve of degree $d \geq 3$ by its generator and minimal relations unless $d=3, n \geq 4$ and $a>0$. Indeed, in this case Lemma 4.4 and Lemma 3.4 show that, for example, also

$$
R /\left(x_{2}, \ldots, x_{n}, x_{1}^{a+n-3}, x_{0}^{a+i} x_{1}^{n-3-i} \mid 1 \leq i \leq n-3\right)
$$

is the Hartshorne-Rao module module of an extremal curve of degree 3 and genus $g_{\max }-a$.

From an Liaison-theoretic point of view minimal curves are particular interesting. In this respect we get.

Remark 4.9. Since every curve $C$ has the property that the function $j \mapsto h^{1}\left(\mathcal{I}_{C}(j)\right)$ is strictly increasing for $j=t, \ldots, 0$ provided $h^{1}\left(\mathcal{I}_{C}(t)\right)>0$ where $t<0$, every extremal curve is minimal in its even Liaison class.

In the next section we will show that extremal curves also have maximal second cohomology.

\section{Properties of extremal Curves}

The construction of the previous section does not provide all extremal curves. In fact, we do not have an explicit description of all such curves. Nevertheless, we are able to derive properties of extremal curves. Our results show that the situation for space curves is more rigid and simpler than for curves of codimension $\geq 3$.

We begin with the computation of the Hartshorne-Rao module.

Theorem 5.1. Let $C \subset \mathbb{P}^{n}$ be a non-degenerate curve of degree $d \geq 3$ and genus $g$. Suppose that at least one of the three conditions

(i) $d=3$,

(ii) $g<g_{\max }=\left(\begin{array}{c}d-2 \\ 2\end{array}\right)-(n-3)$,

(iii) $n \geq 4$ 
is not satisfied. Then $C$ is an extremal curve if and only if its Hartshorne-Rao module is (up to change of coordinates) isomorphic to $R /\left(x_{2}, \ldots, x_{n}, h \cdot\left(x_{0}, x_{1}\right)^{n-3}, f\right)\left(\left(\begin{array}{c}d-2 \\ 2\end{array}\right)-g-1\right)$ where $\operatorname{deg} h=g_{\text {max }}-g, \operatorname{deg} f=\left(\begin{array}{c}d-1 \\ 2\end{array}\right)-g$ and $\left\{x_{2}, \ldots, x_{n}, f, h\right\}$ is a regular sequence if $g<g_{\max }$ and $h=1$ if $g=g_{\max }$.

Remark 5.2. Remark 4.8 shows that the theorem cannot be extended to extremal curves of degree 3 excluded in the statement above.

The proof of Theorem 5.1 requires some preparation.

Lemma 5.3. The Hartshorne-Rao module of an extremal curve is a cyclic R-module.

Proof. If $d=2$ the claim is a consequence of [22], Proposition 3.1.

Now assume $d \geq 3$. Put as before $a:=g_{\max }-g$. We proceed in two steps and use ideas of [17].

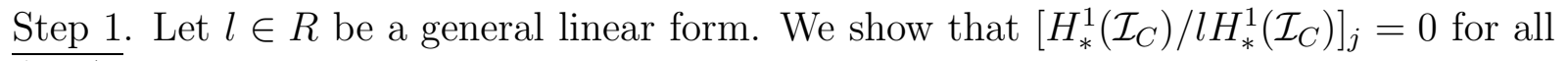
$\bar{j} \geq 1$.

To this end denote by $\Gamma$ the intersection of $C$ and the hyperplane $H$ defined by $l$. Step 1 in the proof of Proposition 2.1 shows $H_{*}^{1}\left(\mathcal{I}_{\Gamma}(j)\right)=0$ if $j \geq d-2$. Since $H_{*}^{1}\left(\mathcal{I}_{C}\right) / l H_{*}^{1}\left(\mathcal{I}_{C}\right)$ is a submodule of $H_{*}^{1}\left(\mathcal{I}_{\Gamma}\right)$, the claim follows for $j \geq d-2$. It remains to consider the integers $j$ with $1 \leq j \leq d-3$.

Since $C$ has maximal cohomology, the inequalities in Steps 2 and 3 of the proof of Proposition 2.1 must be equalities if $1 \leq j \leq d-2$. This provides the claim for $j$ in this range.

Step 2. The Hartshorne-Rao module of $C$ must have the form

$$
H_{*}^{1}\left(\mathcal{I}_{C}\right) \cong(R / \mathfrak{a})(a+n-4)
$$

where $\mathfrak{a}$ is an ideal containing $n-1$ linearly independent forms.

Step 1 provides that the minimal generators of $H_{*}^{1}\left(\mathcal{I}_{C}\right)$ (as $R$-module) have degree $\leq 0$. Thus, the claim is clear if $a+n-3 \leq 1$.

Assume $a+n-3 \geq 2$. The Hilbert function of the $R$-module $H_{*}^{1}\left(\mathcal{I}_{C}\right)$ implies that it has exactly one minimal generator, say $y$, of degree $4-n-a$. Denote by $\mathfrak{a}$ the annihilator of $y$. Then there is an embedding $(R / \mathfrak{a})(a+n-4) \rightarrow H_{*}^{1}\left(\mathcal{I}_{C}\right)$. It follows that $\operatorname{dim}_{K}[R / \mathfrak{a}]_{1} \leq h^{1}\left(\mathcal{I}_{C}(-a-n+5)\right)=2$. Thus, a contains $n-1$ linearly independent forms. Suppose $\mathfrak{a}$ has a further minimal generator $p$ of degree $e \leq a+n-4$. Then we may assume that the hypersurface $P$ defined by $p$ intersects the curve $C$ properly. Hence the exact sequence

$$
0 \rightarrow \mathcal{I}_{C}(-e) \stackrel{p}{\longrightarrow} \mathcal{I}_{C} \rightarrow \mathcal{I}_{C \cap P, P} \rightarrow 0
$$

implies the exact cohomology sequence

$\ldots \rightarrow H^{0}\left(\mathcal{I}_{C \cap P, P}(4-a-n+e)\right) \rightarrow H^{1}\left(\mathcal{I}_{C}(4-a-n)\right) \stackrel{p}{\longrightarrow} H^{1}\left(\mathcal{I}_{C}(4-a-n+e)\right) \rightarrow \ldots$

where the multiplication by $p$ is the zero map. But this gives a contradiction since $4-a-n+e \leq 0$, thus $H^{0}\left(\mathcal{I}_{C \cap P, P}(4-a-n+e)\right)=0$, but $h^{1}\left(\mathcal{I}_{C}(4-a-n)\right)=1$. It follows that the minimal generators of $\mathfrak{a}$ besides the $n-1$ linear forms must have degree $\geq a+n-3$. Comparing vector space dimensions we see that the embedding $(R / \mathfrak{a})(4-n-a) \rightarrow H_{*}^{1}\left(\mathcal{I}_{C}\right)$ is bijective in all degrees $\leq 0$. Therefore, $y$ is the only minimal generator of $H_{*}^{1}\left(\mathcal{I}_{C}\right)$ and the embedding is an isomorphism.

Remark 5.4. By assumption we know the Hilbert function of the Hartshorne-Rao module of an extremal curve. Thus, one might wonder if this information combined with the 
lemma above suffices to determine the module structure. However, this is not the case as shown by the following example:

Consider in the polynomial ring $S=K[x, y]$ the ideals

$$
\mathfrak{a}=\left(x^{a} \cdot(x, y)^{n-3}, y^{b}\right)
$$

and

$$
\mathfrak{b}=\left(x^{a} \cdot(x, y)^{n-3}, x^{a-1} y^{b-a+1}, y^{b+1}\right)
$$

where $a \geq 2$ and $b>a+n-3$. It is easy to see that $S / \mathfrak{a}$ and $S / \mathfrak{b}$ have the same Hilbert function. However Theorem 5.1 says that (up to degree shift) $S / \mathfrak{a}$ is, but $S / \mathfrak{b}$ is not the Hartshorne-Rao module of an extremal curve.

In order to overcome the problem pointed out in Remark 5.4 we compute the generic initial ideal of an extremal curve. Hereby we always are using the reverse lexicographic order on the polynomial ring $R$. It is defined by

$$
\begin{aligned}
x_{0}^{a_{0}} \cdot \ldots \cdot x_{n}^{a_{n}}>x_{0}^{b_{0}} \cdot \ldots \cdot x_{n}^{b_{n}} \text { if the last non-zero coordinate of the vector } & \\
& \left(a_{0}-b_{0}, \ldots, a_{n}-b_{n}, \sum_{i=0}^{n}\left(b_{i}-a_{i}\right)\right)
\end{aligned}
$$

is negative.

We will use various results of the theory of generic initial ideals for which we refer to [9] and [12]. In particular, the generic initial ideals are stable because the ground field $K$ has characteristic zero by assumption.

Proposition 5.5. Let $C \subset \mathbb{P}^{n}$ be an extremal curve of degree $d \geq 3$. Then its generic initial ideal is

$$
\operatorname{gin} I_{C}=\left(x_{0}, \ldots, x_{n-4}\right) \cdot\left(x_{0}, \ldots, x_{n-1}\right)+\left(x_{n-3}^{2}, x_{n-3} x_{n-2}, x_{n-2}^{d}, x_{n-2}^{d-1} x_{n-1}^{a}\right)
$$

where $a:=g_{\max }-g$ and the first summand is defined to be zero if $n=3$, unless $d=3, a \geq 1$ and $n \geq 4$. In the latter case the generic initial ideal of $C$ is either the ideal above or

$$
\left(x_{0}, \ldots, x_{n-5}\right) \cdot\left(x_{0}, \ldots, x_{n-1}\right)+x_{n-4} \cdot\left(x_{0}, \ldots, x_{n-2}\right)+\left(x_{n-3}^{2}, x_{n-3} x_{n-2}, x_{n-2}^{2}, x_{n-4} x_{n-1}^{a+1}\right) .
$$

Proof. Denote by $\Gamma \subset H$ the general hyperplane section of the curve $C$ where $H$ is defined by the linear form $l$. We may assume that $C$ and $\Gamma$ are given in generic coordinates. Thus, their initial ideals agree with their generic initial ideals.

We begin by computing gin $I_{\Gamma} \subset K\left[x_{0}, \ldots, x_{n-1}\right] \cong R / x_{n} R$. By the proof of Proposition 2.1 we know that the Hilbert function of $\Gamma$ is

$$
h_{\Gamma}(j)=\min \{j+2, d\} \quad \text { if } j \geq 1 .
$$

Hence $I_{\Gamma}$ contains exactly $n-3$ linear independent linear forms. By stability of gin $I_{\Gamma}$ we obtain

$$
x_{0}, \ldots, x_{n-4} \in \operatorname{gin} I_{\Gamma} .
$$

Since $I_{\Gamma}$ is saturated $x_{n-1}$ is not a divisor of any minimal generator of gin $I_{\Gamma}$.

Suppose that $d \geq 4$. Then $I_{\Gamma}$ contains all except four quadrics of $R / x_{n} R$. Using stability again we obtain

$$
x_{n-2}^{2}, x_{n-3} x_{n-1}, x_{n-2} x_{n-1}, x_{n-1}^{2} \notin \operatorname{gin} I_{\Gamma} .
$$

If $d=3$ then we get

$$
\left(x_{0}, \ldots, x_{n-4}, x_{n-3}^{2}, x_{n-3} x_{n-2}, x_{n-2}^{2}\right) \subset \operatorname{gin} I_{\Gamma} .
$$

Since both ideals have the same Hilbert function they must be equal. 
Turning again to the case $d \geq 4$ the Hilbert functions of $\Gamma$ implies that $I_{\Gamma}$ has a minimal generator of degree $d-1$. Its initial monomial must be $x_{n-2}^{d-1}$. Comparing Hilbert functions we obtain

$$
\left(x_{0}, \ldots, x_{n-4}, x_{n-3}^{2}, x_{n-3} x_{n-2}, x_{n-2}^{d-1}\right)=\operatorname{gin} I_{\Gamma}
$$

which is also true if $d=3$ as shown above.

In the next step we compute the quadrics contained in gin $I_{C}$. Put $\overline{I_{C}}:=I_{C} / l I_{C}$. By Step 1 in the proof of Lemma 5.3 we have for $j \geq 1$ an exact sequence

$$
0 \rightarrow\left[I_{\Gamma} / \overline{I_{C}}\right]_{j} \rightarrow H^{1}\left(\mathcal{I}_{C}(j-1)\right) \stackrel{l}{\longrightarrow} H^{1}\left(\mathcal{I}_{C}(j)\right) \rightarrow 0
$$

It implies

$$
\operatorname{dim}_{K}\left[I_{\Gamma} / \overline{I_{C}}\right]_{j}= \begin{cases}n-3 & \text { if } j=1 \\ 0 & \text { if } 2 \leq j \leq d-2 \\ 1 & \text { if } d-1 \leq j \leq a+d-2 \\ 0 & \text { if } a+d-1 \leq j\end{cases}
$$

Thus, if $a=0$ then $I_{\Gamma}$ and $\overline{I_{C}}$ agree in all degrees $j \geq 2$. Our claim follows by the knowledge of gin $I_{\Gamma}$.

If $d \geq 4$ and $a \geq 1$ then all the quadrics in $I_{\Gamma}$ are just restrictions of quadrics in $I_{C}$. It follows

$$
\left[\operatorname{gin} I_{C}\right]_{2}=\left[\text { in } I_{C}\right]_{2}=\left[\left(\left(x_{0}, \ldots, x_{n-4}\right) \cdot\left(x_{0}, \ldots, x_{n-1}\right)+\left(x_{n-3}^{2}, x_{n-3} x_{n-2}\right)\right]_{2} .\right.
$$

Using the dimension count $(+)$ we see that gin $I_{C}$ has no further minimal generators in degrees $\leq d-1$, but one in degree $d$ which is in the ideal $x_{n-2}^{d-1} R$. By stability we get $x_{n-2}^{d} \in \operatorname{gin} I_{C}$. Comparing Hilbert functions we see that gin $I_{C}$ has one further minimal generator of degree $d+a-1$ contained in $x_{n-2}^{d-1} R$. Thus, stability provides

$$
\left(x_{0}, \ldots, x_{n-4}\right) \cdot\left(x_{0}, \ldots, x_{n-1}\right)+\left(x_{n-3}^{2}, x_{n-3} x_{n-2}, x_{n-2}^{d}, x_{n-2}^{d-1} x_{n-1}^{a}\right) \subset \operatorname{gin} I_{C} .
$$

Since both ideals have the same Hilbert functions we get equality as claimed.

Finally, consider the case $d=3$ and $a \geq 1$. Then $I_{C}$ contains one quadric less than $I_{\Gamma}$. For reasons of stability it follows that $x_{n-2}^{2}$ or $x_{n-4} x_{n-1}$, provided $n \geq 4$, is the quadric in gin $I_{\Gamma}$ which is not in gin $I_{C}$. In the first case we get as in case $d \geq 4$ that

$$
\operatorname{gin} I_{C}=\left(x_{0}, \ldots, x_{n-4}\right) \cdot\left(x_{0}, \ldots, x_{n-1}\right)+\left(x_{n-3}^{2}, x_{n-3} x_{n-2}, x_{n-2}^{3}, x_{n-2}^{2} x_{n-1}^{a}\right) .
$$

In the second case we conclude by $(+)$ that $x_{n-4} x_{n-1}^{a+1} \in$ gin $I_{C}$. Comparing Hilbert functions we obtain

$$
\begin{aligned}
\operatorname{gin} I_{C}= & \left(x_{0}, \ldots, x_{n-5}\right) \cdot\left(x_{0}, \ldots, x_{n-1}\right)+x_{n-4} \cdot\left(x_{0}, \ldots, x_{n-2}\right) \\
& +\left(x_{n-3}^{2}, x_{n-3} x_{n-2}, x_{n-2}^{2}, x_{n-4} x_{n-1}^{a+1}\right)
\end{aligned}
$$

completing the proof.

We will see in Remark 6.3 that both possibilities for the generic initial ideal of an extremal curves of degree 3 do really occur.

A first consequence of the last result is that extremal curves also have maximal second cohomology. We use the notation of Proposition 2.1.

Corollary 5.6. Let $C \subset \mathbb{P}^{n}$ be an extremal of degree $d \geq 3$. Then we have

$$
h^{2}\left(\mathcal{I}_{C}(j)\right)=\mu_{n}^{e x}(j) \quad \text { for all } j \in \mathbb{Z} \text {. }
$$


Proof. By assumption we know $h^{1}\left(\mathcal{I}_{C}(j)\right)$ and Proposition 5.5 provides the Hilbert function of $C$. Hence, our claim is a consequence of the Riemann-Roch formula

$$
h_{C}(j)-p_{C}(j)=-h^{1}\left(\mathcal{I}_{C}(j)\right)+h^{2}\left(\mathcal{I}_{C}(j)\right) .
$$

Remark 5.7. (i) An analogous statement is also true for extremal curves of degree 2 after modifying the definition of $\mu_{n}^{e x}$. In case $d=2$ we have to put $\mu_{n}^{e x}(j)=0$ if $j \geq 0$. We leave the details to the reader.

(ii) The last results extends part of [7], Lemma 4.8 which also shows that the converse of Corollary 5.6 is true provided $n=3$ and $d \geq 5$. However, the curves in Example 4.6 show that in case $n \geq 4$ there are curves of arbitrarily large degree for which the converse of Corollary 5.6 is false.

As the final preparation for the proof of Theorem 5.1 we compute the Betti numbers of the ideals encountered in Proposition 5.5.

Lemma 5.8. For $1 \leq i \leq n$ define integers

$$
\begin{gathered}
\alpha_{i}=(n-3)\left(\begin{array}{c}
n \\
i
\end{array}\right)+\left(\begin{array}{c}
n-1 \\
i
\end{array}\right)-\left(\begin{array}{c}
n-2 \\
i+1
\end{array}\right) \\
\alpha_{i}^{\prime}=\alpha_{i}-\left(\begin{array}{c}
n-2 \\
i-2
\end{array}\right), \quad \beta_{i}=\left(\begin{array}{c}
n-2 \\
i-1
\end{array}\right), \quad \gamma_{i}=\left(\begin{array}{c}
n-1 \\
i-1
\end{array}\right) .
\end{gathered}
$$

(a) Let $a \geq 0, d \geq 3$ be integers. The ideal

$$
I=\left(x_{0}, \ldots, x_{n-4}\right) \cdot\left(x_{0}, \ldots, x_{n-1}\right)+\left(x_{n-3}^{2}, x_{n-3} x_{n-2}, x_{n-2}^{d}, x_{n-2}^{d-1} x_{n-1}^{a}\right)
$$

has a minimal free resolution of the form

$$
0 \rightarrow R^{n-3}(-n-1) \oplus R(-d-a-n+2) \rightarrow F_{n-1} \rightarrow \ldots \rightarrow F_{1} \rightarrow I \rightarrow 0
$$

where if $a>0$

$$
F_{i}=R^{\alpha_{i}}(-i-1) \oplus R^{\beta_{i}}(-i-d+1) \oplus R^{\gamma_{i}}(-i-d-a+2) \quad(1 \leq i \leq n-1)
$$

and if $a=0$

$$
F_{i}=R^{\alpha_{i}}(-i-1) \oplus R^{\beta_{i}}(-i-d+2) \quad(1 \leq i \leq n-1) .
$$

(b) For $n \geq 4$ and $a>0$ the minimal free resolution of the ideal $J=\left(x_{0}, \ldots, x_{n-5}\right) \cdot\left(x_{0}, \ldots, x_{n-1}\right)+x_{n-4} \cdot\left(x_{0}, \ldots, x_{n-2}\right)+\left(x_{n-3}^{2}, x_{n-3} x_{n-2}, x_{n-2}^{2}, x_{n-4} x_{n-1}^{a+1}\right)$. has the shape

$$
0 \rightarrow R^{n-4}(-n-1) \oplus R(-a-n-1) \rightarrow G_{n-1} \rightarrow \ldots \rightarrow G_{1} \rightarrow J \rightarrow 0
$$

where

$$
G_{i}=R^{\alpha_{i}^{\prime}}(-i-1) \oplus R^{\gamma_{i}}(-i-a-1) \quad(1 \leq i \leq n-1) .
$$

Proof. Observe that the two ideals are stable. Thus their minimal free resolution is described in a theorem of Eliahou and Kervaire [10]. A straightforward, but lengthy computation provides the graded Betti numbers using the formula in [12, Corollary 1.32 and the identity

$$
\sum_{k=i+1}^{n-3}(k+1)\left(\begin{array}{c}
k \\
i-1
\end{array}\right)=(n-2)\left(\begin{array}{c}
n-2 \\
i
\end{array}\right)-\left(\begin{array}{c}
n-2 \\
i+1
\end{array}\right)
$$

Combining the information we have obtained we finally can determine the structure of the Hartshorne-Rao module of extremal curves. 
Proof of Theorem 5.1. The other implication being clear it suffices to show that the Hartshorne-Rao module of an extremal curve $C \subset \mathbb{P}^{n}$ (with admissible $d, g, n$ ) is

$$
H_{*}^{1}\left(\mathcal{I}_{C}\right) \cong R /\left(x_{2}, \ldots, x_{n}, h \cdot\left(x_{0}, x_{1}\right)^{n-3}, f\right)(4-n-a)
$$

where $a=g_{\max }-g$.

Using the notation of Step 2 in the proof of Lemma 5.3 we have

$$
R / \mathfrak{a} \cong S / \mathfrak{b}
$$

where $\mathfrak{b}$ is an ideal in the polynomial ring $S=K[x, y] \cong R /\left(x_{2}, \ldots, x_{n}\right) R$. Thus, it remains to determine the ideal $\mathfrak{b}$. We use the knowledge of the Hilbert function of $S / \mathfrak{b}$. If $a=0$ we immediately get $\mathfrak{b}=(x, y)^{n-3}$ as claimed. Thus, we may assume $a \geq 1$ for the rest of the proof. Then the Hilbert function of $S / \mathfrak{b}$ still implies two facts:

(i) $\mathfrak{b}$ has $n-2$ minimal generators of degree $a+n-3$ which have $n-3$ linear syzygies,

(ii) $\mathfrak{b}$ has a syzygy of degree $2 a+d+n-5$.

Now we use a result of Rao (cf. [28], Theorem 2.5 and its generalization [20], Proposition 5.6, respectively). Combined with the Cancellation principle (cf., e.g., [12], Corollary 1.21) we obtain that $\operatorname{Tor}_{n+1}^{R}\left(H_{*}^{1}\left(\mathcal{I}_{C}\right), K\right)$ is a direct summand of $\operatorname{Tor}_{n}^{R}\left(R / \operatorname{gin} I_{C}, K\right)$. Therefore the comparison with Lemma 5.8 shows that the syzygies of $\mathfrak{b}$ pointed out in (i), (ii) above are the only syzygies of $\mathfrak{b}$. Hence $\mathfrak{b}$ has besides the $n-2$ minimal generators of degree $a+n-3$ exactly one more generator $f$ which has degree $a+d+n-5$.

Finally, the fact that the Hilbert function of $S / \mathfrak{b}$ has maximal growth in degree $a+n-2$ implies that the $n-2$ minimal generators of degree $a+n-3$ of $\mathfrak{b}$ have a common divisor, say $h$, of degree $a$. It follows that

$$
\mathfrak{b}=\left(h \cdot(x, y)^{n-3}, f\right) .
$$

Since $S / \mathfrak{b}$ has finite length $f, h$ must be a regular sequence as claimed.

Remark 5.9. Although we excluded all extremal curves of degree 2 and many of degree 3 in Theorem 5.1 there is still some information on the Hartshorne-Rao module of such curves.

(i) Using the notation of the proof above its arguments provide for extremal curves with $d=3, a \geq 1$ and $n \geq 4$ that $S / \mathfrak{b}$ must have a free resolution of the form

$0 \rightarrow S^{n-3}(-a-n+2) \oplus S(-2 a-n+2) \rightarrow S^{n-2}(-a-n+3) \oplus S(-a-n+2) \rightarrow S \rightarrow S / \mathfrak{b} \rightarrow 0$

which is possibly not minimal. The latter case can really occur as Remark 4.8 shows.

(ii) A similar description is also possible for extremal curves $C \subset \mathbb{P}^{n}$ of degree 2 . Indeed, Proposition 3.1 in 22] implies that then the Hartshorne-Rao module is

$$
H_{*}^{1}\left(\mathcal{I}_{C}\right) \cong S / \mathfrak{b}(-g-1)
$$

where $S / \mathfrak{b}$ has a minimal free resolution of the form

$$
0 \rightarrow S^{n-3}(g-1) \oplus S(2 g+n-3) \rightarrow S^{n-1}(g) \rightarrow S \rightarrow S / \mathfrak{b} \rightarrow 0 .
$$

The next goal is to show that extremal curves most often contain a planar subcurve of largest possible degree.

Proposition 5.10. Let $C \subset \mathbb{P}^{n}$ be an extremal curve satisfying either

(i) $d \geq 5$ or

(ii) $d=4$ and $g<g_{\max }$.

Then $C$ contains a planar subcurve of degree $d-1$. 
Proof. In case (i) the claim follows by [6], Corollary 4.4. However, we will give a selfcontained proof of our stronger assertion.

Define the subscheme $Z \subset \mathbb{P}^{n}$ as the intersection of the quadrics containing the curve $C$. We may assume that $C$ and $Z$ are given in generic coordinates. Then we claim that every minimal basis of the ideal $I_{Z}$ is in fact a Gröbner basis. Indeed, all the degree three Spolynomials of the generators of $I_{Z}$ reduce to zero because Proposition 5.5 shows that the elements of the Gröbner basis of $I_{C}$ have either degree 2 or a degree $\geq \min \{d, d-1+a\}>3$.

Using Proposition 5.5 again it follows that the generic initial ideal of $Z$ is

$$
\operatorname{gin} I_{Z}=\left(x_{0}, \ldots, x_{n-4}\right) \cdot\left(x_{0}, \ldots, x_{n-1}\right)+\left(x_{n-3}^{2}, x_{n-3} x_{n-2}\right) .
$$

Computing the Hilbert polynomial of $R / \operatorname{gin} I_{Z}$ we see that $Z$ is a two-dimensional scheme of degree 1 , in other words $Z$ is the union of a plane, $P$, and a scheme of dimension $\leq 1$. We also may assume that $Z$ is in generic coordinates. Thus, we have

$$
\operatorname{gin} I_{Z}=\left(x_{0}, \ldots, x_{n-3}\right) \text {. }
$$

Hence we obtain

$$
\operatorname{in}\left(I_{C} \cap I_{P}\right) \subset \operatorname{in} I_{C} \cap \operatorname{in} I_{P}=\operatorname{gin} I_{C} \cap \operatorname{gin} I_{P}=\operatorname{gin} I_{Z}=\text { in } I_{Z} .
$$

Since we clearly have $I_{Z} \subset I_{C} \cap I_{P}$ it follows

$$
I_{Z}=I_{C} \cap I_{P}
$$

Using the exact sequence

$$
0 \rightarrow R / I_{Z} \rightarrow R / I_{C} \oplus R / I_{P} \rightarrow R /\left(I_{C}+I_{P}\right) \rightarrow 0
$$

we see that $I_{C}+I_{P}$ defines a one-dimensional scheme which is the union of a planar curve of degree $d-1$ and possibly a zero-dimensional scheme. Our assertion follows.

Remark 5.11. Ellia [11 has shown that a non-degenerate curve $C \subset \mathbb{P}^{3}$ of degree $d \geq 5$ is extremal if and only if $C$ contains a planar subcurve of degree $d-1$. However, the converse of Proposition 5.10 is not true if $n \geq 4$. There are non-degenerate curves in $\mathbb{P}^{n}, n \geq 4$, of arbitrarily large degree $d$ which do contain a planar subcurve of degree $d-1$, but whose cohomology is smaller than the one of extremal curves (cf. Example 4.6).

\section{BetTi Numbers of EXTREMAL CURVES}

Lemma 5.8 provides upper bounds for the graded Betti numbers of an extremal curve $C$. The next result shows that actually these bounds are attained in most cases. The key will be the existence of another suitable subcurve of $C$.

Proposition 6.1. Adopt the notation of Lemma 5.8. Let $C \subset \mathbb{P}^{n}$ be an extremal curve satisfying either

(i) $d \geq 5 \quad$ or

(ii) $d=4$ and $g<g_{\max }$.

Then the minimal free resolution of $C$ has the form

$$
0 \rightarrow R^{n-3}(-n-1) \oplus R(-d-a-n+2) \rightarrow F_{n-1} \rightarrow \ldots \rightarrow F_{1} \rightarrow I \rightarrow 0
$$

where in case $a=g_{\max }-g>0$

$$
F_{i}=R^{\alpha_{i}}(-i-1) \oplus R^{\beta_{i}}(-i-d+1) \oplus R^{\gamma_{i}}(-i-d-a+2) \quad(1 \leq i \leq n-1)
$$

and if $a=0$

$$
F_{i}=R^{\alpha_{i}}(-i-1) \oplus R^{\beta_{i}}(-i-d+2) \quad(1 \leq i \leq n-1)
$$


Proof. The Cancellation principle combined with Lemma 5.8 shows that $C$ has a free resolution as claimed. We have to show its minimality. This is obvious if $a=0$ because then our assumption implies $d \geq 5$. Thus it remains to consider the case $a \geq 1$.

Assuming that $C$ is in generic coordinates Proposition 5.5 shows that the Gröbner basis of $I_{C}$ consists of quadrics and two forms $g_{1}, g_{2}$ with

$$
\text { in } g_{1}=x_{n-2}^{d} \quad \text { and } \quad \text { in } g_{2}=x_{n-2}^{d-1} x_{n-1}^{a} \text {. }
$$

Using the notation of the proof of Proposition 5.10 define the ideal $J \subset R$ by

$$
J:=I_{Z}+g_{1} R=\left[I_{C}\right]_{2} R+g_{1} R .
$$

Since $I_{C} \cap I_{P}=I_{Z}$ we obtain

$$
J \cap I_{P}=I_{Z} \subset I_{P}
$$

It follows that $J+I_{P}=I_{P}+g_{1} R$ defines a planar curve of degree $d$. Using the exact sequence

$$
0 \rightarrow R / I_{Z} \rightarrow R / J \oplus R / I_{P} \rightarrow R /\left(I_{P}+g_{1} R\right) \rightarrow 0
$$

we see that $J$ has the same Hilbert function as $\operatorname{gin}\left(I_{Z}\right)+x_{n-2}^{d} R$. Since obviously $\operatorname{gin}\left(I_{Z}\right)+$ $x_{n-2}^{d} R \subset$ in $J$ we conclude

$$
\text { in } J=\operatorname{gin}\left(I_{Z}\right)+x_{n-2}^{d} R .
$$

Now in $(J)$ has a minimal free resolution of the form

$$
0 \rightarrow R^{n-3}(-n-1) \rightarrow F_{n-1} \rightarrow \ldots \rightarrow F_{1} \rightarrow I \rightarrow 0
$$

where, using the notation of Lemma 5.8,

$$
F_{i}=R^{\alpha_{i}}(-i-1) \oplus R^{\beta_{i}}(-i-d+1) \quad(1 \leq i \leq n-1) .
$$

Since $d \geq 4$ any resolution of this shape must be minimal. Thus, the graded Betti numbers of $J$ and in $(J)$ agree. Observing that the minimal free resolution of $J$ injects into the one of $I_{C}$ we see that the resolution described in the statement is minimal as claimed.

Recall that an ideal $I \subset R$ is said to be componentwise linear if the ideal $[I]_{j} R$ has a linear free resolution for all integers $j$. Our last result implies.

Corollary 6.2. The homogeneous ideal of an extremal curve satisfying either

(i) $d \geq 5$ or

(ii) $d=4$ and $g<g_{\max }$.

is componentwise linear.

Proof. Let $C$ be an extremal curve satisfying our assumption. Combining Proposition 5.5, Lemma 5.8 and Proposition 6.1 we see that the graded Betti numbers of $I_{C}$ and gin $I_{C}$ agree. Hence the main result of [四] implies our claim.

Finally, we show that Proposition 5.5 can not improved in general.

Remark 6.3. We want to show that in case $d=3, n \geq 4, a \geq 2$ the two possiblities for the generic initial ideal of an extremal curve allowed by Proposition 5.5 do really occur. Indeed, the generic initial ideal of the corresponding curve in Example 4.5 is

$$
\left(x_{0}, \ldots, x_{n-4}\right) \cdot\left(x_{0}, \ldots, x_{n-1}\right)+\left(x_{n-3}^{2}, x_{n-3} x_{n-2}, x_{n-2}^{3}, x_{n-2}^{2} x_{n-1}^{a}\right) .
$$

This follows by comparing the graded Betti numbers of the curve and the ideals in Lemma 5.8 using the same arguments as in the proof of Theorem 5.1.

Now consider the curve $C$ defined by the ideal

$$
I_{C}=\left(x_{2}, \ldots, x_{n}\right)^{2}+\left(x_{0}^{a+1} x_{3}+x_{1}^{a+1} x_{4}, x_{0} x_{i}+x_{1} x_{i+1} \mid 4 \leq i \leq n-1\right) .
$$


Using Construction 3.3 we see that $C$ is an extremal curve of degree 3 and genus $g_{\max }-a$. The ideal $I_{C}$ is componentwise linear and

$$
\operatorname{gin} I_{C}=\left(x_{0}, \ldots, x_{n-5}\right) \cdot x_{n-1}+\left(x_{0}, \ldots, x_{n-2}\right)^{2}+\left(x_{n-4} x_{n-1}^{a+1}\right) .
$$

The methods developed in this paper are rather general. Thus, we believe that they can be succesfully applied to other related problems.

\section{REFERENCES}

[1] A. Aramova, J. Herzog, T. Hibi, Ideals with stable Betti numbers, Adv. Math. 152 (2000), 72-77.

[2] E. Ballico, N. Chiarli, S. Greco, Linearly normal curves with degenerate general hyperplane section, Hiroshima Math. J. (to appear).

[3] M. Brodmann, Bounds on the cohomological Hilbert functions of a projective variety, J. Algebra 109 (1987), 352-380.

[4] M. Brodmann, U. Nagel, Bounding cohomological Hilbert functions by hyperplane sections, J. Algebra 174 (1995), 323-348.

[5] N. Chiarli, S. Greco, U. Nagel, On the genus and Hartshorne-Rao module of projective curves, Math. Z. 229 (1998), 695-724.

[6] N. Chiarli, S. Greco, U. Nagel, When does a projective curve contain a planar subcurve?, J. Pure Appl. Algebra 164 (2001), 345-364.

[7] N. Chiarli, S. Greco, U. Nagel, Surfaces in $\mathbb{P}^{4}$ with extremal general hyperplane section, J. Algebra (to appear).

[8] N. Chiarli, S. Greco, U. Nagel, Families of space curves with large cohomology (in preparation).

[9] D. Eisenbud, "Commutative algebra with a view toward algebraic geometry," Graduate Texts in Mathematics 150, Springer-Verlag, 1995.

[10] S. Eliahou, M. Kervaire, Minimal resolutions of some monomial ideals, J. Algebra 129 (1990), 1-25.

[11] Ph. Ellia, On the cohomology of projective space curves, Boll. Un. Mat. Ital. (7) 9-A (1995), 593-607.

[12] M. Green, Generic initial ideals, In: "Six lectures on commmutative algebra" (J. Elias, J. M. Giral, R. M. Miro-Roig, S. Zarzuela, Eds.), Progress in Mathematics 166, Birkhäuser, 1998, pp. 119-185.

[13] R. Hartshorne, The genus of space curves, Ann. Univ. Ferrara-Sez VII-Sc. Mat. 40 (1994), 207-223, 1996.

[14] C. Huneke, B. Ulrich, General hyperplane sections of algebraic varieties, J. Algebraic Geom. 2 (1993), 487-505.

[15] M. Martin-Deschamps, D. Perrin, Sur la classification des courbes gauches, Astérisque 184-185 (1990).

[16] M. Martin-Deschamps, D. Perrin, Sur les bornes du module de Rao, C. R. Acad. Sci. Paris 317 (1993), 1159-1162.

[17] M. Martin-Deschamps, D. Perrin, Le schema de Hilbert de courbes localement de Cohen-Macaulay n'est (presque) jamais reduit, Ann. Sci. Ec. Norm. Sup 29 (1996), 757-785.

[18] J. Migliore, On linking double lines, Trans. Amer. Math. Soc. 294 (1986), 177-185.

[19] J. Migliore, "Introduction to Liaison theory and deficiency modules," Progress in Mathematics 165, Birkhäuser, 1998.

[20] J. Migliore, U. Nagel, C. Peterson, Buchsbaum-Rim sheaves and their multiple sections, J. Algebra 219 (1999), 378-420.

[21] J. Migliore, U. Nagel and C. Peterson, Constructing schemes with prescribed cohomology in arbitrary codimension, J. Pure Appl. Algebra 152 (2000), 245-251.

[22] U. Nagel, R. Notari, M. L. Spreafico, Curves of degree two and ropes on a line: their ideals and even liaison classes, Preprint, 2002.

[23] U. Nagel, On bounds for cohomological Hilbert functions, J. Algebra 150 (1992), 231-244.

[24] U. Nagel, On the cohomology and genus of projective curves, Le Matematiche, Special volume in honor of Silvio Greco (to appear).

[25] S. Nollet, Subextremal curves, Manuscripta Math. 94 (1997), 303-317.

[26] R. Notari, M. L. Spreafico, On curves of $\mathbb{P}^{n}$ with extremal Hartshorne-Rao module in positive degrees, J. Pure Appl. Algebra 156 (2001), 95-114.

[27] C. Okonek, H. Spindler, Das Spektrum torisionsfreier Garben, II, Springer Lecture Notes in Math. 1165 (1985), 211-234. 
[28] A. P. Rao, Liaison among curves in $\mathbb{P}^{3}$, Invent. Math. 50 (1979), 205-217.

[29] T. Sauer, Nonstable reflexive sheaves on $\mathbb{P}^{3}$, Trans. Amer. Math. Soc. 281 (1984), 633-655.

[30] R. Strano, A characterization of complete intersections in $\mathbb{P}^{3}$, Proc. Amer. Math. Soc. 104 (1988), $711-715$. 